\title{
THE ONSET CONDITIONS OF OSCILLATORY MOTION OF SINGLE GAS BUBBLES RISING IN VARIOUS LIQUIDS
}

\author{
HIDEKI TSUGE AND SHIN-ICHI HIBINO \\ Department of Applied Chemistry, Keio Gijuku University, Yokohama 223
}

Many studies have been made about the behaviors of single gas bubbles ascending in various liquids, that is, the rising velocities or the shapes of single bubbles ${ }^{2,4,8,9)}$. Single bubbles rise in a straight line when the bubble diameter is very small, and with increase of the bubble diameter, the path of the gas bubble turns to be zigzag or spiral.

On the condition of onset of zigzag or spiral motion, Saffman $^{6)}$ and Hartunian and Sears ${ }^{3)}$ carried out experimental and theoretical work. Saffman ${ }^{6)}$ suggested that the zigzag motion was caused by the interaction between a periodic oscillation of the wake and the instability of the motion near the front of the bubble, and deduced that the value of the critical Weber number $W e_{c}$ at which instability appeared was 1.03. But this criterion does not agree with experimental results.

Hartunian and Sears ${ }^{3 !}$ suggested that the interaction between surface tension and hydrodynamic pressure could result in instability of the bubble motion, and on a basis of the existing data and their own experimental results, they proposed that there were two distinct criteria for the instability:

1) for the impure and somewhat more viscous liquids, a critical Reynolds number $R e_{c}=202$;

2) for the pure, relatively low viscous liquids, a critical Weber number $W e_{c}=3.18$.

But in their work the relations between the onset conditions of oscillation and physical properties of liquids are not clear.

The objects of this study are to determine experimentally the onset conditions of oscillation of single gas bubbles rising in various liquids and to propose a more general criterion for the onset conditions of oscillation.

\section{Experimental}

The experimental apparatus was almost the same one used for the previous work ${ }^{9}$. The bubble column made of pyrex glass of $80 \mathrm{~mm}$ in diameter was installed on a brass base by sealing with purified mercury. In order to prevent the liquid from contamination, the

Received July 20, 1976. Correspondence concerning this article should be addressed to $H$. Tsuge. liquid was in contact only with glass, metal and purified mercury. Glass square tank was set to reduce the optical distortion in case of photographing.

The desired volume of air was injected with a microsyringe, which was previously calibrated, into the bubble generation device and the air bubble was formed slowly from the glass nozzle in the liquid.

When the bubble ascended about $15 \mathrm{~cm}$ above the glass nozzle, the bubble was photographed against a black background by using a stroboscopic flash bulb placed above the liquid surface. The frequency of flash was $2600-3000$ cycles $/ \mathrm{min}$.

10 bubbles of the same equivalent spherical diameter were photographed and the average rising velocity was determined. When the paths of more than 5 bubbles of the same equivalent spherical diameter taken on photographs were zigzag, its diameter and average rising velocity were considered as the critical diameter and the critical rising velocity.

Table 1 shows the liquids used for experiments and their physical properties were measured. The organic liquids used for experiments were reagent grade, and the distilled water was used in preparing each aqueous solution.

\section{Experimental Results and Discussions}

With the increase of the bubble diameter, the ascending path of single gas bubbles changes from a straight line to an oscillatory path. The experimental results, that is, the critical diameter $d_{c}$, rising velocity $U_{c}$, Reynolds number $R e_{c}$ and Weber number $W e_{c}$ at which oscillatory motion appears are slso shown in Table 1.

It is known that the motion of bubble ascending in a liquid is influenced considerably by the purity of liquid $^{4,9}$. But, since the quantitative representation of the purity of liquid is not yet clear, whether the liquid used was pure or not was judged from the comparison of the shape of $C_{D}$ vs. $R e$ curve obtained in this experiment with that of the literatures ${ }^{4,8)}$. As the result, the liquids used for this experiment except distilled water are considered to belong to the purified system. Distilled water showed an intermediate behavior between the purified system and the contaminated system. 
Table 1 Physical properties of liquids and critical values of onset of bubble oscillation

\begin{tabular}{|c|c|c|c|c|c|c|c|c|c|}
\hline Liquid & $\begin{array}{c}\text { Temp. } \\
{\left[{ }^{\circ} \mathrm{C}\right]}\end{array}$ & {$\left[\mathrm{g} / \mathrm{cm}^{\mathrm{s}}\right]$} & $\stackrel{\mu}{\mathrm{cP}]}$ & $\begin{array}{c}\sigma \\
\text { [dyne } / \mathrm{cm}]\end{array}$ & $\stackrel{M}{[-]}$ & $\begin{array}{c}d_{c} \\
{[\mathrm{~cm}]}\end{array}$ & $\begin{array}{c}U_{c} \\
{[\mathrm{~cm} / \mathrm{sec}]}\end{array}$ & $\begin{array}{l}R e_{c} \\
{[-]}\end{array}$ & $\begin{array}{l}W e_{c} \\
{[-]}\end{array}$ \\
\hline Distilled water & 21.0 & 0.998 & 0.981 & 72.5 & $2.39 \times 10^{-11}$ & 0.171 & 22.8 & 397 & 1.22 \\
\hline Methanol & 22.2 & 0.784 & 0.618 & 22.5 & $1.60 \times 10^{-10}$ & 0.124 & 29.1 & 458 & 3.66 \\
\hline $60 \%$ Methanol in water & 22.3 & 0.894 & 1.50 & 31.3 & $1.81 \times 10^{-9}$ & 0.250 & 21.6 & 321 & 3.31 \\
\hline $30 \%$ Methanol in water & 22.2 & 0.951 & 1.69 & 41.0 & $1.22 \times 10^{-8}$ & 0.250 & 23.4 & 329 & 3.17 \\
\hline $46 \%$ Glycerine in water & 22.0 & 1.115 & 4.50 & 68.1 & $1.14 \times 10^{-8}$ & 0.292 & 25.5 & 185 & 3.11 \\
\hline $48 \%$ Glycerine in water & 20.0 & 1.110 & 5.19 & 67.7 & $2.07 \times 10^{-8}$ & 0.319 & 26.0 & 178 & 3.54 \\
\hline $50 \%$ Glycerine in water & 23.0 & 1.125 & 5.21 & 66.2 & $2.21 \times 10^{-8}$ & 0.325 & 25.9 & 182 & 3.70 \\
\hline $52 \%$ Glycerine in water & 19.0 & 1.132 & 6.51 & 67.2 & $5.13 \times 10^{-8}$ & 0.363 & 26.6 & 168 & 4.33 \\
\hline Isoamyl alcohol & 22.4 & 0.809 & 4.11 & 23.0 & $2.82 \times 10^{-7}$ & 0.350 & 19.8 & 136 & 4.80 \\
\hline $60 \%$ Soybean oil in hexane & 21.3 & 0.825 & 5.33 & 25.4 & $5.85 \times 10^{-7}$ & 0.337 & 21.0 & 110 & 4.85 \\
\hline
\end{tabular}

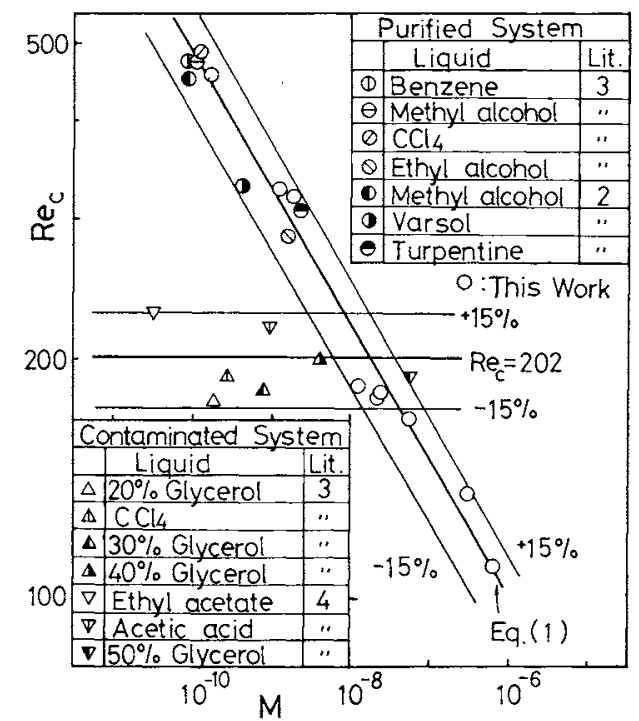

Fig. 1 Relation between $R e_{c}$ and $M$ for purified and contaminated systems

To clarify the relation between the condition of onset of oscillation and the physical properties of liquids, $R e_{c}$ and dimensionless group consisted of physical properties of liquid $M$ are plotted in Fig. 1. $R e_{c}$ is correlated within an accuracy of $\pm 15 \%$ for the purified system as follows,

$$
R e_{o}=9.0 M^{-0.173}
$$

$R e_{c}$ are plotted against $M$ using the literature data which are considered to belong to the purified system and they are well correlated with Eq. (1) as shown in Fig. 1.

$W e_{c}$ and $R e_{c}$ of the foregoing data for the purified system are plotted in Fig. 2 as proposed by Hartunian et $a l .^{3)}$. We is correlated within an accuracy of $\pm 25 \%$ as follows,

$$
W e_{c}=21.5 R e_{c}^{-0.32}
$$

By the above comparison, it can be said that Eq. (1) or (2) is applicable for wider range of $M$, that is, 8.4 . $10^{-11}<M<5.9 \cdot 10^{-7}$ than the criterion suggested by Hartunian et al..$^{3}$.

The relations of $R e_{c}$ and $M$ for contaminated system

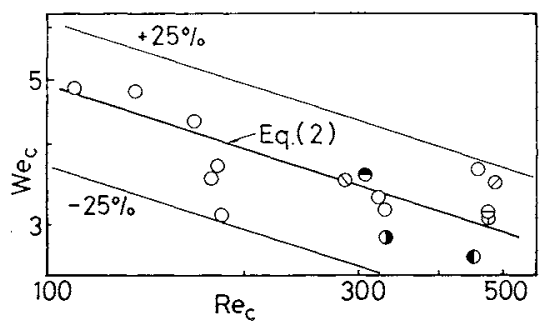

Fig. $2 W e_{c}$ vs. $R e_{c}$ for purified system. The keys are same as in Fig. 1

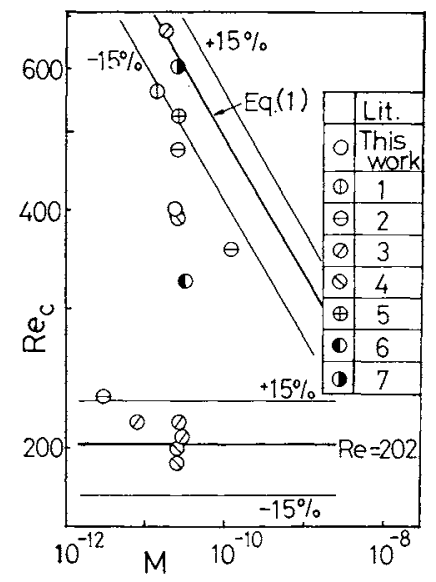

Fig. 3 Relation between $R e_{c}$ and $M$ for water

in the existing literatures are also plotted in Fig. 1. The criterion proposed by Hartunian et al..$^{3}$, that is, $R e_{0}=202$ is applicable to the liquids of the contaminated system independent of $M$ in the range $3.3 \cdot 10^{-11}$ $<M<5.5 \cdot 10^{-8}$.

Water is a peculiar liquid in the sense that it is very sensitive to contamination. It has been pointed out that the rising velocities of single bubbles ascending in the water scatter considerably ${ }^{10}$.

In Fig. $3 R e_{c}$ for water obtained in this experiment is plotted against $M$ together with other researchers' data. Though some points are well represented by Eq. (1), and points for tap water and contaminated water lie near the line $R e_{c}=202$, it can be said that the values of $R e_{c}$ scatter between two lines. 


\section{Acknowledgment}

The authors are indebted to Mr. T. Hiraishi and Mr. S. Sasaki for their experimental assistance.

Nomenclature

$C_{D} \quad=$ drag coefficient of bubble, $4 d g / 3 U^{2}$

$d \quad=$ equivalent spherical diameter of bubble $\quad[\mathrm{cm}]$

$g \quad=$ gravitational acceleration $\quad\left[\mathrm{cm} / \mathrm{sec}^{2}\right]$

$M \quad=$ dimensionless parameter, $g \mu^{4} / \rho \sigma^{3}$

$\operatorname{Re} \quad=$ Reynolds number, $d U \rho / \mu$ [-]

$U \quad=$ terminal velocity of bubble $\quad[\mathrm{cm} / \mathrm{sec}]$

We = Weber number, $d U^{2} \rho / \sigma \quad[-]$

$\mu \quad=$ liquid viscosity $\quad[\mathrm{g} / \mathrm{cm} \cdot \mathrm{sec}]$

$\rho \quad=$ liquid density $\quad\left[\mathrm{g} / \mathrm{cm}^{3}\right]$

$\sigma \quad=$ liquid surface tension $\quad[\mathrm{dyne} / \mathrm{cm}]$

$\langle$ Subscript $\rangle$

$c \quad=$ critical

\section{Literature Cited}

1) Aybers, N. M. and A. Tapucu: Wärme- $u$. Stoffübertragung, 2, 118 (1969).

2) Haberman, W. L. and R. K. Morton: David Taylor Model Basin, Report 802 (1953).

3) Hartunian, R. A. and W. R. Sears: J. Fluid Mech., 3, 27 (1957).

4) Kubota, M., T. Akehata and T. Shirai: Kagaku Kögaku, 31, 1074 (1967).

5) Rosenberg, B.: David Taylor Model Basin, Report 727 (1950).

6) Saffman, P. G.: J. Fluid Mech., 1, 249 (1956).

7) Stuke, B.: Naturwissenschaften, 39, 325 (1952).

8) Tadaki, T. and S. Maeda: Kagaku Kögaku, 25, 254 (1961).

9) Tsuge, H. and S. Hibino: ibid., 35, 65 (1971).

10) Tsuge, H., M. Tasaki and S. Hibino: ibid., 35, 1263 (1971).

\title{
MOTION OF DROPS IN NON-NEWTONIAN FLUID SYSTEMS AT LOW REYNOLDS NUMBER
}

\author{
YoshinoRI KAWASE and Yasuo HIROSE \\ Department of Industrial Chemistry, Tokyo Metropolitan University, Tokyo 158
}

In previous work ${ }^{97}$, motion and mass transfer of fluid spheres in non-Newtonian fluid systems were analyzed for the high Reynolds number region. The proposed theory agreed well with experimental data available in the literature. The purpose of this paper is to investigate motion of drops in non-Newtonian fluid systems for the low Reynolds number region. A semiempirical equation for the drag coefficient of drops in non-Newtonian fluid systems is presented.

For characterization of the non-Newtonian behavior of fluids, the power law model is used.

$$
\tau_{j}^{i}=-K\left(\frac{1}{2} \Delta_{k}^{m} \Delta_{k}^{m}\right)^{\langle n-1\} / 2} \Delta_{j}^{i}
$$

We consider that the drag coefficient of drops in non-Newtonian fluid systems is analogous to that in Newtonian fluid systems.

For the low Reynolds number region, the drag coefficient of drops in Newtonian fluid systems is given $\mathrm{as}^{2,8)}$

$$
C_{D_{N}}=\frac{24}{R e_{N}} \frac{2 X_{N}+3}{3 X_{N}+3}
$$

where

Received September 1, 1976. Correspondence concerning this article should be addressed to $Y$. Kawase.

$$
R e_{N}=\rho d U / \mu \text { and } X_{N}=\mu / \mu^{\prime}
$$

When the Reynolds number and the viscosity ratio in Eq. (2) are respectively replaced with those in power law fluid systems given by

$$
R e_{N N}=\rho d^{n} U^{2-n} / K
$$

and

$$
X_{N N}=\left(\frac{K U^{n-1}}{d^{n-1}}\right) /\left(\begin{array}{c}
K^{\prime} U^{n^{\prime}-1} \\
d^{n^{\prime}-1}
\end{array}\right)
$$

we consider that the drag coefficient of drops in power law fluid systems is approximately predicted by Eq. (2). The Reynolds number in power law fluid systems as defined Eq. (3) is often used to discuss motion of non-Newtonian fluid ${ }^{6,12)}$. The viscosity ratio defined as Eq. (4) is used by Marrucci et al. ${ }^{3)}$ and Shirotsuka and Kawase ${ }^{10)}$. When motion of Newtonian drops in non-Newtonian fluids is discussed, Eq. (4) becomes $K / \mu^{\prime} \cdot(U / d)^{n-1}$ which was used by some investigators ${ }^{6)}$. Of course, when both external and internal fluids are Newtonian fluids, Eqs. (3) and (4) become the Reynolds number and the viscosity ratio in Newtonian fluid systems defined as Eq. (2), respectively.

The relationship of the ratio of the drag coefficient of drops in non-Newtonian fluid systems to that in Newtonian fluid systems and the non-Newtonian behavior of external fluids is shown in Fig. 1. A 